Discrete Comput Geom 31:197-206 (2004)

DOI: $10.1007 / \mathrm{s} 00454-003-0805-8$

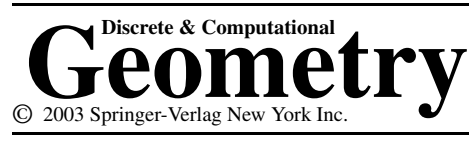

\title{
Acute Triangulations of the Regular Icosahedral Surface*
}

\author{
Jin-ichi Itoh ${ }^{1}$ and Tudor Zamfirescu ${ }^{2}$ \\ ${ }^{1}$ Faculty of Education, Kumamoto University, \\ Kumamoto 860, Japan \\ j-itoh@gpo.kumamoto-u.ac.jp \\ ${ }^{2}$ Fachbereich Mathematik, Universität Dortmund, \\ 44221 Dortmund, Germany \\ tudor.zamfirescu@mathematik.uni-dortmund.de
}

\begin{abstract}
We prove here that the surface of the regular icosahedron can be triangulated with 8 non-obtuse and with 12 acute triangles. We also show these numbers to be smallest possible.
\end{abstract}

\section{Introduction}

In 1953 MacNeal showed interest in non-obtuse triangulations as they appeared in connection with the discretization of partial differential equations [17].

The discussion of acute triangulations has one of its origins in a problem of Stover reported in 1960 by Gardner in his Mathematical Games section of the Scientific American (see [8] and [9]). There the question was raised whether a triangle with one obtuse angle can be cut into smaller triangles, all of them acute. In the same year, independently, Burago and Zalgaller [2] investigated in considerable depth acute triangulations of polygonal complexes, being led to them by the problem of their isometric embedding into $\mathbb{R}^{3}$. (Accidentally, their paper also includes a solution to Stover's problem!)

Motivated by the proof of the discrete maximum principle, in 1973 Ciarlet and Raviart [4] and Strang and Fix [21], and later Santos [20], were also led to non-obtuse triangulations.

In 1980 Cassidy and Lord [3] considered acute triangulations of the square. Recently, Maehara investigated acute triangulations of quadrilaterals [18] and other polygons [19].

* The first author was partially supported by a Grant-in-Aid for Scientific Research, the Ministry of Education, Culture, Sports, Science and Technology, Japan. Also partially supported by JSPS and DAAD at the University of Dortmund, Germany, 2002. The second author was partially supported by JSPS at Kumamoto University, Japan, 2002. 
Acute triangulations with triangles which are close to equilateral were considered by Gerver [10] and, on Riemannian surfaces, by Colin de Verdière and Marin [6]. Also, Baker et al. [1] investigated non-obtuse triangulations of polygons. Extensions to three dimensions were considered by Křížek and Qun [14], Korotov and Křížek [15] and Korotov et al. [16].

A triangulation of a two-dimensional space means a collection of (full) triangles covering the space, such that the intersection of any two triangles is either empty or consists of a vertex or of an edge. A triangle is called geodesic if all its edges are segments, i.e., shortest paths between the corresponding vertices. We are interested only in geodesic triangulations, all the members of which are, by definition, geodesic triangles.

Colin de Verdière [5] shows how to transform a triangulation of a compact surface of non-positive curvature into a geodesic triangulation. The planar case was previously treated by Wagner [23] (see also [7] and [22]). From now on, triangulation will always mean a geodesic one.

Our interest is focused on triangulations which are non-obtuse or acute, which means that the angles of all appearing geodesic triangles are not larger than, respectively smaller than, $\pi / 2$.

We started together with Hangan in [11] the investigation of acute triangulations of all Platonic surfaces, which are the surfaces of the five well-known Platonic solids.

For the regular tetrahedron and octahedron, their natural triangulation is optimal in the sense that it contains the smallest number of triangles. Among the remaining non-trivial cases only the cube was treated completely [11]. This study was continued for the case of the regular icosahedron by Itoh [12], who provided triangulations with $n$ triangles for all even numbers $n \geq 16$. Also, in [13], we treated the case of the regular dodecahedron, completely settled the non-obtuse case and found a surprisingly small acute triangulation (with 14 triangles only). The question whether a triangulation with 12 acute triangles does or does not exist is still open.

Here we treat the case of the regular icosahedron. We regard our work as a small step towards a solution to the following problem first raised in [11]. We consider this problem very natural, and far from trivial.

Problem 1. Does there exist a number $N$ such that every compact convex surface in $\mathbb{R}^{3}$ admits an acute triangulation with at most $N$ triangles?

Of course, Problem 1 can be extended (or restricted) to other families of surfaces (such as Riemannian), with or without boundary. Even more generally, families $\mathcal{F}$ of twodimensional triangulable compact topological spaces may be considered. In particular, $\mathcal{F}$ can consist of two-dimensional compact Alexandrov spaces with a common lower (or upper) bound for the curvature. Even the very particular family of all tetrahedral surfaces seems to be quite interesting.

In the previous paper [11] the following problem was formulated.

Problem 2. Find the minimal number of triangles of a non-obtuse, respectively acute, triangulation of the Platonic surfaces in the non-trivial cases, i.e., for the surface of the cube, of the regular dodecahedron and of the regular icosahedron. 
In [11] we proved together with Hangan that the surface of a cube admits several acute triangulations with 24 triangles, and no acute triangulation with fewer triangles. What about its non-obtuse triangulations?

The surface of a cube admits a non-obtuse triangulations with four triangles! Indeed, if we denote the vertices of the upper square by $a, b, c, d$ with $a$ and $c$ non-adjacent, and denote the vertex of the bottom square which is adjacent to $a$ (resp. $b, c, d$ ) by $a^{\prime}$ (resp. $\left.b^{\prime}, c^{\prime}, d^{\prime}\right)$, then the four equilateral triangles $b d a^{\prime}, b d c^{\prime}, a^{\prime} c^{\prime} b, a^{\prime} c^{\prime} d$ form a non-obtuse triangulation.

Clearly, each triangulation of any two-dimensional manifold has an even number of triangles.

In this paper we completely settle both questions about the minimal non-obtuse and the minimal acute triangulation of the regular icosahedral surface. We construct a highly asymmetrical triangulation with 12 triangles and are convinced that a (minimal) example possessing any symmetry does not exist.

\section{Non-Obtuse Triangulations}

Theorem 1. The surface of the regular icosahedron admits a non-obtuse triangulation with eight triangles and no non-obtuse triangulation with fewer triangles.

Proof. Figure 1 describes the surface of a regular icosahedron (on the left-hand side the upper half of ten equilateral triangles, on the right-hand side the lower half of ten equilateral triangles). Take a face $a b c$ of the left-hand side of Fig. 1. Let $a^{\prime}$ (resp. $b^{\prime}, c^{\prime}$ ) be the antipodal vertex of $a$ (resp. $b, c$ ). Draw the segments from $a$ (resp. $b$ and $c$ ) to $b^{\prime}, c^{\prime}$ (resp. $c^{\prime}, a^{\prime}$ and $a^{\prime}, b^{\prime}$ ). We get the non-obtuse triangles

$$
a b c, a^{\prime} b^{\prime} c^{\prime}, a b^{\prime} c^{\prime}, b c^{\prime} a^{\prime}, c a^{\prime} b^{\prime}, a^{\prime} b c, b^{\prime} c a, c^{\prime} a b .
$$

Indeed, $a b c$ and $a^{\prime} b^{\prime} c^{\prime}$ are equilateral and planar. In the case of the triangle $a b^{\prime} c^{\prime}$, there are two right angles at $b^{\prime}$ and $c^{\prime}$, while $\angle b^{\prime} a c^{\prime}=\pi / 3$. The remaining triangles are all congruent to $a b^{\prime} c^{\prime}$. Hence all eight triangles are non-obtuse.

We prove now that eight is the smallest possible number of non-obtuse triangles. The only triangulations of the sphere with less than eight triangles are $K_{4}$ and the 1-skeleton of the double pyramid over a triangle. In both cases there are vertices with degree 3 . If
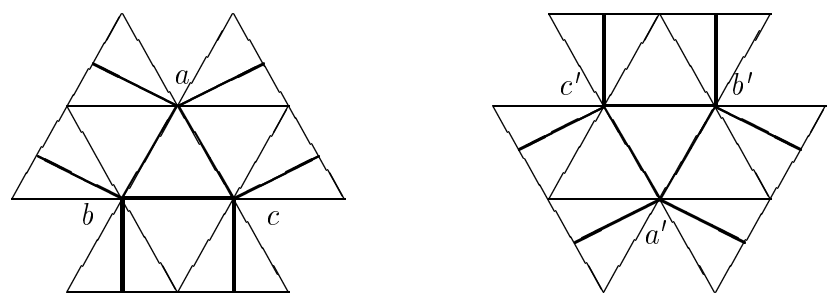

Fig. 1 
the triangulation is non-obtuse, at such a vertex the total angle around it is at most $3 \pi / 2$. However, at each vertex of the icosahedron the total angle is $5 \pi / 3>3 \pi / 2$. Hence there are no non-obtuse triangulations of the icosahedron with less than eight triangles.

\section{Acute Triangulations with Few Triangles}

Let $\mathcal{I}$ be the 1-skeleton of the regular icosahedron. The graph-theoretic distance $d_{\mathcal{I}}(v, w)$ between vertices $v, w$ of $\mathcal{I}$ is called the $\mathcal{I}$-distance. The intrinsic distance on the surface between two points $p, q$ is denoted by $|p q|$.

Theorem 2. The regular icosahedral surface admits an acute triangulations with 12 triangles, and no acute triangulation with fewer triangles.

Proof ( first part). Assume the edges of the icosahedron have length 1. We present an acute triangulation consisting of 12 triangles.

First, we fix an antipodal pair of vertices $a, b$ and a vertex $a^{\prime}$ adjacent in $\mathcal{I}$ to $a$. Take a vertex $b^{\prime}$ adjacent to $b$, at $\mathcal{I}$-distance 2 from both $a$ and $a^{\prime}$. Denote (consecutively) the other vertices around $a$ by $a_{1}, a_{2}, a_{3}, a_{4}$. Also, denote the other vertices around $b$ by $b_{1}, b_{2}, b_{3}, b_{4}$, such that $b_{1}$ is adjacent to $a_{2}, a_{3}$ (see Fig. 2).

Let $d$ (resp. $c^{\prime}$ ) be the midpoint of the line-segment joining the midpoints of $b_{1} a_{2}$ (resp. $a_{1} b_{2}$ ) and $b_{1} a_{3}$ (resp. $a_{1} b_{3}$ ). Denote the midpoint of $b^{\prime} a_{4}$ by $x$ and the midpoint of $a_{3} a_{4}$ by $y$. Let $c$ be the midpoint of the line-segment joining $x$ with the midpoint $c^{+}$ of $x y$. Let $z$ be the midpoint of $b_{3} b_{4}$. Take the point $d^{\prime}$ on $b_{4} z$ such that $\angle d^{\prime} a^{\prime} c^{\prime}=\pi / 2$. It will be shown later that $\angle d^{\prime} b b_{4}<\pi / 12, \angle b d^{\prime} c^{\prime}<\pi / 2$, and the segments $a^{\prime} b_{4}$ and $c d^{\prime}$ are orthogonal, whence $\angle c d^{\prime} a^{\prime}<\pi / 2$. We choose a point $d^{*}$ on $d^{\prime} z$ close to $d^{\prime}$, such that $\angle d^{*} b b_{4}<\pi / 12, \angle b d^{*} c^{\prime}<\pi / 2$ and $\angle c d^{*} a^{\prime}<\pi / 2$ too. Note that $\angle d^{*} a^{\prime} c^{\prime}<\angle d^{\prime} a^{\prime} c^{\prime}=\pi / 2$ and $\angle d^{*} b c^{\prime}<\angle b 4 b c^{\prime}=\pi / 2$. Take a point $c^{*}$ on $c^{\prime} a_{1}$ close to $c^{\prime}$, such that still $\angle d^{*} a^{\prime} c^{*}<\pi / 2$ and $\angle d^{*} b c^{*}<\pi / 2$. More conditions about how close $c^{*}$ and $c^{\prime}$ must be appear later.

We got a triangulation with 12 triangles:

$$
a a^{\prime} c, a a^{\prime} c^{*}, a c d, a c^{*} d, a^{\prime} c d^{*}, a^{\prime} c^{*} d^{*}, b b^{\prime} d, b b^{\prime} d^{*}, b^{\prime} c d, b^{\prime} c d^{*}, b c^{*} d, b c^{*} d^{*} .
$$

There are two shortest paths from $b$ to $d$; here we chose the path crossing $b_{1} b_{2}$.

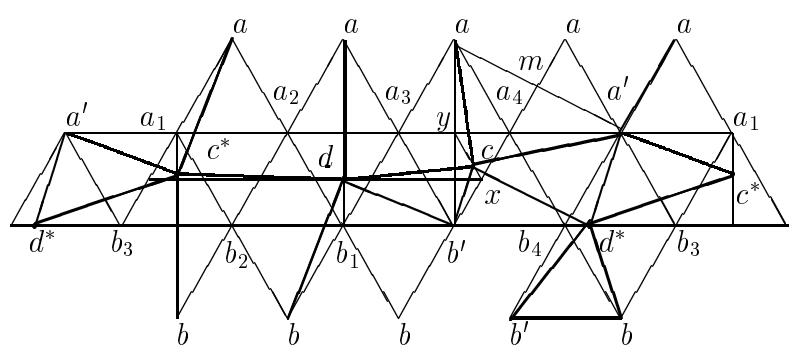

Fig. 2 
We show that all these triangles are acute.

First note that, in the plane $\Pi$ on which the icosahedron is unfolded in Fig. 2, the circle $C$ of centre $m$ and radius $\sqrt{3} / 2$ passes through $y$. (The point $m \in \Pi$ is shown in Fig. 2.) Since, in $\Pi$, the angle $x y m$ is right, the line-segment $x y$ is tangent to $C$ at $y$. This fact yields $\angle a t a^{\prime}<\pi / 2$ not only in $\Pi$ but on the icosahedron too, for every point $t \in c^{+} x$. In particular, $\angle a c a^{\prime}<\pi / 2, \angle a c^{+} a^{\prime}<\pi / 2$ (for any choice of the segment $c^{+} a^{\prime}$ ) and, since $\left\{a, c^{+}, a^{\prime}\right\}$ and $\left\{a^{\prime}, c^{\prime}, a\right\}$ are congruent, $\angle a^{\prime} c^{\prime} a<\pi / 2$ (for any choice of the segment $\left.a c^{\prime}\right)$. When choosing the point $c^{*}$ we arrange that $\angle a^{\prime} c^{*} a<\pi / 2$ too. Since $d^{*}$ lies between $z$ and $b_{4}, \angle b_{4} a^{\prime} d^{*}<\pi / 6$ and $\angle c a^{\prime} d^{*}<\angle a_{4} a^{\prime} z=\pi / 2$. From the construction, $\angle d^{*} a^{\prime} c^{*}<\pi / 2$ and $\angle a a^{\prime} c^{*}<\angle a a^{\prime} c^{\prime}<\pi / 2$. Moreover, $\angle d a c^{*}<\angle d a a_{1}=\pi / 2$, $\angle d a c<\angle d a a_{4}=\pi / 2, \angle c^{*} a a^{\prime}<\angle b_{2} a a^{\prime}=\pi / 2, \angle c a a^{\prime}<\angle b^{\prime} a a^{\prime}=\pi / 2$ and $\angle c a^{\prime} a<\angle b^{\prime} a^{\prime} a=\pi / 2$. Around $a$ and $a^{\prime}$ we have checked all angles.

Next we consider the angles around $b$ and $b^{\prime}$. It is clear that $\angle d^{*} b^{\prime} b<\pi / 3, \angle d b b^{\prime}<$ $\angle a_{2} b b^{\prime}=\pi / 2, \angle d^{*} b b^{\prime}<\angle a^{\prime} b b^{\prime}=\pi / 2, \angle d b c^{*}<\angle b_{1} b c^{\prime}=\pi / 2$ and $\angle d b^{\prime} b<$ $\angle a_{2} b^{\prime} b=\pi / 2$, while $\angle d^{*} b c^{*}<\pi / 2$ by construction.

Since $|c x| /|c y|=\frac{1}{4}$ but $\left|x b^{\prime}\right| /\left|y b^{\prime}\right|=1 / \sqrt{3}>\frac{1}{4}$, we have $\angle x b^{\prime} c<\angle y b^{\prime} c$. Thus, $\angle a_{4} b^{\prime} c<\pi / 12$. Moreover, $\angle b^{\prime} a x<\angle a_{4} a x$. Hence

$$
\angle d^{\prime} a^{\prime} b_{4}=\angle c^{\prime} a^{\prime} b_{2}=\angle c^{+} a b^{\prime}<\angle b^{\prime} a x<\frac{\pi}{12} .
$$

Obviously, $\angle d^{\prime} b^{\prime} b_{4}<\angle d^{\prime} a^{\prime} b_{4}$, whence $\angle d^{\prime} b b_{4}<\pi / 12$ and, by construction, $\angle d^{*} b b_{4}<$ $\pi / 12$ too. Hence

$$
\angle c b^{\prime} d^{*}=\angle c b^{\prime} a_{4}+\frac{\pi}{3}+\angle d^{*} b^{\prime} b_{4}<\frac{\pi}{2} .
$$

Let $p$ and $q$ denote the midpoints of $b_{1} a_{3}$ and $b^{\prime} a_{3}$ respectively, and $\{r\}=b^{\prime} d \cap b_{1} a_{3}$ (see Fig. 3). Ceva's theorem with respect to the triangle $a_{3} p q$ and the line through $b^{\prime}$ and $d$ gives $|p r|=\frac{1}{10}$, whence $\tan \angle d b^{\prime} a_{2}=\sqrt{3} / 15$. If $c^{-}$is the orthogonal projection

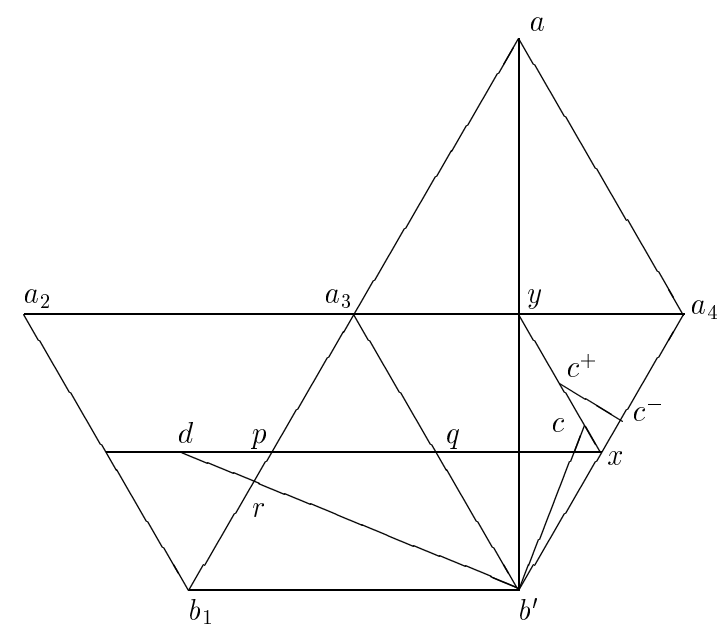

Fig. 3 
of $c^{+}$on $a_{4} b^{\prime}$, then $\left|c^{+} c^{-}\right|=\sqrt{3} / 8$ and $\left|b^{\prime} c^{-}\right|=\frac{5}{8}$. Thus,

$$
\tan \angle a_{4} b^{\prime} c>\frac{\left|c^{+} c^{-}\right| / 2}{\left|b^{\prime} c^{-}\right|}=\frac{\sqrt{3}}{10} .
$$

Hence $\angle a_{4} b^{\prime} c>\angle d b^{\prime} a_{2}$, which yields $\angle c b^{\prime} d<\pi / 2$.

Consider now the angles around $d$. From the choice of $c^{*}$ it follows that $\angle a d c^{*}<$ $\angle a d c^{\prime}=\pi / 2$. Further, $\angle a d c<\angle a d x=\pi / 2$. Also, $\angle b^{\prime} d b=\angle a c^{\prime} a^{\prime}<\pi / 2$, as shown previously. It is clear that $\angle b d c^{\prime}<\pi / 2$. By taking $c^{*}$ close enough to $c^{\prime}$, we assure $\angle b d c^{*}<\pi / 2$.

Obviously, $\angle b^{\prime} d c$ is smaller than the angle between $a_{3} b^{\prime}$ and $d c$, which is smaller than the angle between $a_{3} b^{\prime}$ and $b_{1} c$, which is smaller than the right angle between $a_{3} b^{\prime}$ and $b_{1} a_{4}$.

We pass to the angles around $c$. We have

$$
\tan \angle c d x=\frac{|c g|}{|d g|}=\frac{\sqrt{3}}{19},
$$

where $g$ is the orthogonal projection of $c$ on $d x$. Also,

$$
\tan \angle c a b^{\prime}=\frac{|c f|}{|f a|}>\frac{\sqrt{3}}{12},
$$

where $f$ is the orthogonal projection of $c$ on $b^{\prime} a$ (see Fig. 4). Hence $\angle c d x<\angle c a b^{\prime}$, which implies $\angle a c d<\pi / 2$.

We already saw that $\angle a c a^{\prime}<\pi / 2$. It is clear that $\angle a^{\prime} c d^{*}<\angle a^{\prime} c b_{4}<\pi / 3$.

Let $e$ be the intersection of the line through $f$ and $c$ with the perpendicular to $a_{4} a^{\prime}$ through $d^{\prime}$. Simple calculations show that $\left|b^{\prime} f\right|=\left|d^{\prime} e\right|=5 \sqrt{3} / 16,|f c|=\frac{3}{16},\left|d^{\prime} z\right|=\frac{3}{8}$

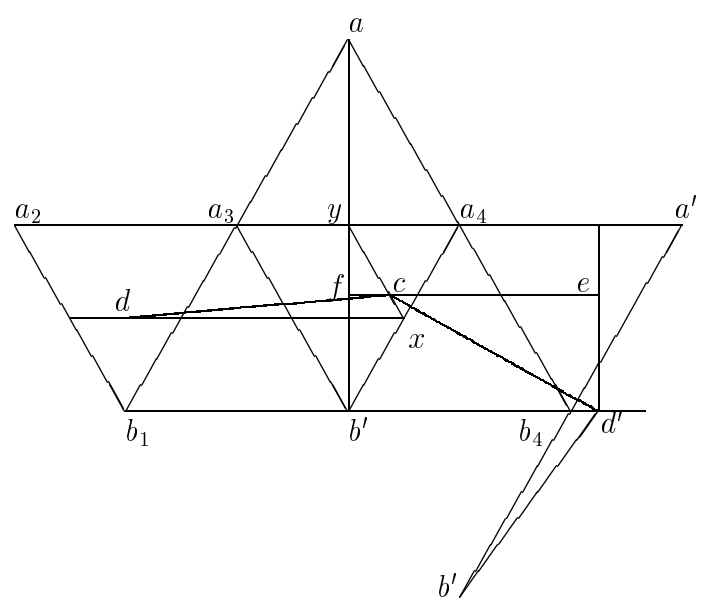

Fig. 4 
and $|c e|=\frac{15}{16}$. Hence

$$
\tan \angle c b^{\prime} f=\frac{\sqrt{3}}{5}<\frac{\sqrt{3}}{3}=\tan \angle e c d^{\prime},
$$

which implies $\angle b^{\prime} c d^{\prime}<\pi / 2$. It is clear that $\angle b^{\prime} c d<\pi / 2$.

Now consider the angles around $c^{*}$. The angle $a c^{*} a^{\prime}$ was shown to be acute. Also, $\angle a c^{\prime} d<\angle a_{1} c d=\pi / 2$, and if $c^{*}$ is close enough to $c^{\prime}$, then $\angle a c^{*} d<\pi / 2$ too. Clearly, $\angle d c^{*} b<\angle d c^{\prime} b=\pi / 2$ and

$$
\angle b c^{*} d^{*}<\angle b c^{\prime} d^{*}<\angle b c^{\prime} b_{4}<\frac{\pi}{2} .
$$

Because $\left|a^{\prime} c^{*}\right|>\left|a^{\prime} a_{1}\right|>\left|a^{\prime} d^{*}\right|$, we have $\angle a^{\prime} d^{*} c^{*}>\angle a^{\prime} c^{*} d^{*}$, whence $\angle a^{\prime} c^{*} d^{*}<$ $\pi / 2$.

Finally, we consider the angles around $d^{*}$. It is clear that $\angle a^{\prime} d^{*} c^{*}<\angle a^{\prime} d^{*} b_{3}<\pi / 2$. We found above that $\tan \angle e c d^{\prime}=\sqrt{3} / 3$, which means that $\angle e c d^{\prime}=\pi / 6$. Hence $d^{\prime} c$ is orthogonal to $a^{\prime} b_{4}$; therefore $\angle b^{\prime} d^{\prime} c<\pi / 2$. Also, $\angle b^{\prime} d^{*} c<\angle b^{\prime} d^{\prime} c<\pi / 2$. It is obvious that $\angle b d^{*} b^{\prime}<\angle b b_{4} b^{\prime}=\pi / 3$.

Let $v, w$ denote the midpoints of $b_{3} a_{1}, b_{3} a^{\prime}$ respectively (see Fig. 5). We already found $\left|d^{\prime} z\right|=\frac{3}{8}$. Hence

$$
\left|b_{4} d^{\prime}\right|=\frac{1}{8}<\frac{1}{4}=\left|v c^{\prime}\right| .
$$

It follows that the angle $\alpha$ between $b_{4} w$ and $d^{\prime} c^{\prime}$ is larger than $\angle w b_{4} v$. We also already found that $\tan \angle d b^{\prime} a_{2}=\sqrt{3} / 15$. Moreover,

$$
\angle d b^{\prime} a_{2}=\angle c^{\prime} a^{\prime} v=\angle b_{4} a^{\prime} d^{\prime}=\angle b_{4} b d^{\prime}
$$

and $\tan \angle w b_{4} v=\sqrt{3} / 9$. So,

$$
\alpha>\angle w b_{4} v>\angle d b^{\prime} a_{2}=\angle b_{4} b d^{\prime} .
$$

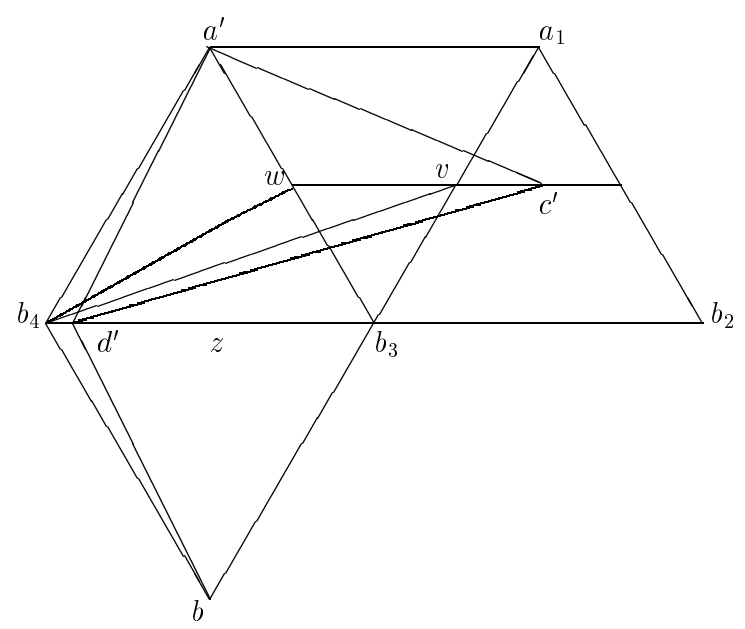

Fig. 5 
Hence $\angle b d^{\prime} c^{\prime}<\pi / 2$. Now, our initial construction guarantees that $\angle b d^{*} c^{\prime}<\pi / 2$ too. Finally, by construction, $\angle c d^{*} a^{\prime}<\pi / 2$.

We finished the proof that all triangles are acute.

\section{No Acute Triangulation with Even Fewer Triangles}

We start by proving the following lemma.

Lemma 1. Suppose $a, b, b^{\prime}$ are vertices of the acute triangulation $\mathcal{T}$ of the regular icosahedral surface, such that a has degree 4 in $\mathcal{T}, b b^{\prime}$ is an edge of $\mathcal{I}$ but not of $\mathcal{T}$ and $b, b^{\prime}$ are neighbours of $a$ in $\mathcal{T}$. Then $\angle a b b^{\prime}>2 \pi / 3$ and $\angle a b^{\prime} b>2 \pi / 3$.

Proof. The above vertices $b, a, b^{\prime}$ of $\mathcal{T}$ form a path of length 2 in $\mathcal{T}$.

Since the degree of $a$ in $\mathcal{T}$ is $4, a$ is a vertex of $\mathcal{I}$. Because $a b$ and $a b^{\prime}$ are nonconsecutive edges of $\mathcal{T}$ at $a$ and $\mathcal{T}$ is acute, necessarily $\angle b a b^{\prime}>2 \pi / 3$.

After fixing the edge $b b^{\prime}$, we look for the possible location of $a$.

If $d_{\mathcal{I}}(a, b)=d_{\mathcal{I}}\left(a, b^{\prime}\right)=1$, then $\angle b a b^{\prime}=\pi / 3$, which is bad. If $d_{\mathcal{I}}(a, b)=2$ and $d_{\mathcal{I}}\left(a, b^{\prime}\right)=1$, then $\angle b a b^{\prime}=\pi / 6$, even worse. If $d_{\mathcal{I}}(a, b)=d_{\mathcal{I}}\left(a, b^{\prime}\right)=2$, then $\angle b a b^{\prime}=\pi / 3$, bad again.

The only remaining case (essentially) is $d_{\mathcal{I}}(a, b)=3$ and $d_{\mathcal{I}}\left(a, b^{\prime}\right)=2$, when $a$ and $b$ are opposite vertices of the icosahedron. Looking at Fig. 2 we find points $a, b, b^{\prime}$ in such a position. There are ten segments from $a$ to $b$. By choosing one of those two closest to $a_{1}$ as $a b$, we indeed have $\angle b a b^{\prime}>2 \pi / 3$, for all the other eight this inequality fails to be true. Then $\angle a b b^{\prime}>2 \pi / 3$ and $\angle a b^{\prime} b=5 \pi / 6>2 \pi / 3$ too.

Proof of Theorem 2 (last part). By Theorem 1, we only have to show that there is no acute triangulation of the regular icosahedral surface with eight or ten triangles.

Suppose there exists an acute triangulation $\mathcal{T}$ of the regular icosahedral surface containing eight triangles. The only triangulation of the sphere with eight triangles and degree at least 4 at every vertex is the 1 -skeleton of the regular octhedron. All its vertices have degree 4 . Therefore all vertices of $\mathcal{T}$ are vertices of $\mathcal{I}$.

It is easily seen that any acute angle $p q r$ between two segments $p q$ and $q r$ joining vertices of the icosahedron is at most $\pi / 3$ or equals $\angle b b_{1} x$ or equals $\angle a b_{1} x$ (see Fig. 2). The second and third values appear only if $q$ is antipodal either to $p$ or to $r$.

Let $p_{0}$ be a vertex of $\mathcal{T}$. Because the triangulation is acute, among the four angles around $p_{0}$ at most one can be equal to or less than $\pi / 3$. Further, at most two among the angles around $p_{0}$ can take the second or third value mentioned above, because at most one of the four distinct neighbours of $p_{0}$ can be antipodal to $p_{0}$. Since there is a fourth angle at $p_{0}$, a contradiction is obtained.

Suppose now that a triangulation $\mathcal{T}$ with ten acute triangles exists. The only triangulation of the sphere with ten triangles and degree at least 4 at every vertex is the 1-skeleton of the double pyramid over the pentagon. Let $C_{5}$ be the 5-cycle in $\mathcal{T}$ containing all 4 -valent vertices. Clearly, the vertices of $C_{5}$ must be vertices of $\mathcal{I}$.

We claim that there are two vertices $p_{1}, p_{2}$ of $C_{5}$ such that $p_{1} p_{2}$ is an edge of $\mathcal{I}$. 
Indeed, suppose this is not true. Then all five neighbours in $\mathcal{I}$ of $p_{1} \in C_{5}$ do not belong to $C_{5}$. Among all five vertices at $\mathcal{I}$-distance 2 from $p_{1}$ there are at most two vertices in $C_{5}$. Now, only one vertex is left, while $C_{5}$ contains five vertices! So, the claim is proved.

We further claim that there are three vertices $p_{1}, p_{2}, p_{3} \in C_{5}$ such that $p_{1} p_{2}$ and $p_{2} p_{3}$ are edges of $\mathcal{I}$. Indeed, we already found an edge $p_{1} p_{2}$ of $\mathcal{I}$ with $p_{1}, p_{2} \in C_{5}$, but suppose the new claim is wrong. Then the six vertices of $\mathcal{I}$ at $\mathcal{I}$-distance 1 from $\left\{p_{1}, p_{2}\right\}$ are not in $C_{5}$. So the remaining $K_{4}$ minus one edge must contain the other three vertices of $C_{5}$, which is impossible without contradicting the assumption. Also the second claim is true.

Clearly, $p_{1} p_{2}$ and $p_{2} p_{3}$ cannot both be edges of $\mathcal{T}$, because if they were, then $\angle p_{1} p_{2} p_{3} \leq 2 \pi / 3$ and $\mathcal{T}$ would not be acute.

The cycle $C_{5}$ contains two more vertices, $p_{4}$ and $p_{5}$. So there are (essentially) two cases concerning the order on $C_{5}: p_{1}, p_{4}, p_{2}, p_{5}, p_{3}$ and $p_{1}, p_{2}, p_{4}, p_{3}, p_{5}$. Also, $d_{\mathcal{I}}\left(p_{1}, p_{3}\right)$ may be 1 or 2 .

Case $p_{1}, p_{4}, p_{2}, p_{5}, p_{3}$. The presence of the segment $p_{1} p_{3}$ implies

$$
\angle p_{1} p_{2} p_{4}+\angle p_{4} p_{2} p_{5}+\angle p_{5} p_{2} p_{3} \leq \frac{4 \pi}{3} .
$$

(If $d_{\mathcal{I}}\left(p_{1}, p_{3}\right)=1$, the sum is $4 \pi / 3$, if $d_{\mathcal{I}}\left(p_{1}, p_{3}\right)=2$, the sum is $\pi$.) However, $\angle p_{1} p_{2} p_{4}>2 \pi / 3$ and $\angle p_{5} p_{2} p_{3}>2 \pi / 3$ by Lemma 1 , and a contradiction is obtained.

Case $p_{1}, p_{2}, p_{4}, p_{3}, p_{5}$. We have

$$
\angle p_{1} p_{2} p_{4}+\angle p_{4} p_{2} p_{3} \leq \frac{4 \pi}{3} .
$$

(If $d_{\mathcal{I}}\left(p_{1}, p_{3}\right)=1$, the sum is $\pi / 3$ or $4 \pi / 3$, if $d_{\mathcal{I}}\left(p_{1}, p_{3}\right)=2$, the sum is $2 \pi / 3$ or $\pi$.) However, $\angle p_{1} p_{2} p_{4}>2 \pi / 3$ because $\mathcal{T}$ is acute, and $\angle p_{4} p_{2} p_{3}>2 \pi / 3$ by Lemma 1 . A contradiction is obtained again.

\section{References}

1. B. S. Baker, E. Grosse, C. S. Rafferty, Nonobtuse triangulation of polygons, Discrete Comput. Geom., 3 (1988), 147-168

2. Y. D. Burago, V. A. Zalgaller, Polyhedral embedding of a net (Russian), Vestnik Leningrad. Univ., 15 (1960), 66-80.

3. Ch. Cassidy, G. Lord, A square acutely triangulated, J. Recreational Math., 13 (1980-81), 263-268.

4. P. G. Ciarlet, P. A. Raviart, Maximum principle and uniform convergence for the finite element method, Comput. Methods Appl. Mech. Engrg., 2 (1973), 17-31.

5. Y. Colin de Verdière, Comment rendre géodésique une triangulation d'une surface? Enseign. Math. (2), 37 (1991), 201-212.

6. Y. Colin de Verdière, A. Marin, Triangulations presque équilaterales des surfaces, J. Differential Geom., 32 (1990), 199-207.

7. I. Fary, On straight line representation of planar graphs, Acta Sci. Math. Szeged, 11 (1948), 229-233.

8. M. Gardner, Mathematical games. A fifth collection of "brain-teasers", Scientific American, 202(2) (1960), 150-154.

9. M. Gardner, Mathematical games. The games and puzzles of Lewis Carroll, and the answers to February's problems, Scientific American, 202(3) (1960), 172-182. 
10. J. L. Gerver, The dissection of a polygon into nearly equilateral triangles, Geom. Dedicata, 16 (1984), 93-106.

11. T. Hangan, J. Itoh, T. Zamfirescu, Acute triangulations, Bull. Math. Soc. Sci. Math. Roumanie, 43 (2000), 279-286.

12. J. Itoh, Acute triangulations of sphere and icosahedron, Josai Math. Monographs, 3 (2001), 53-61.

13. J. Itoh, T. Zamfirescu, Acute triangulations of the regular dodecahedral surface, to appear.

14. M. Kř́žzek, L. Qun, On diagonal dominance of stiffness matrices in 3D, East-West J. Numer. Math., 3(1) (1995), 59-69.

15. S. Korotov, M. Kř́žzek, Acute type refinements of tetrahedral partitions of polyhedral domains, manuscript, 2001.

16. S. Korotov, M. Křǐžek, P. Neittaanmäki, Weakened acute type condition for tetrahedral triangulations and the discrete maximum principle, Report B 11/1999, University of Jyväskylä.

17. R. H. MacNeal, An asymmetrical finite difference network, Quart. Appl. Math., 11 (1953), 295-310.

18. H. Maehara, On acute triangulations of quadrilaterals, Proc. JCDCG 2000, to appear.

19. H. Maehara, Acute triangulations of polygons, European J. Combin., 23 (2002), 45-55.

20. V. R. Santos, On the strong maximum principle for some piecewise linear finite element approximate problems of non-positive type, J. Fac. Sci. Univ. Tokyo Sect. IA Math., 29 (1982), 473-491.

21. G. Strang, G. J. Fix, An Analysis of the Finite Element Method, Prentice-Hall, Englewood Cliffs, NJ, 1973.

22. W. Tutte, How to draw a graph, Proc. London Math. Soc., 13 (1963), 743-768.

23. K. Wagner, Bemerkungen zum Vierfarbenproblem, Jahresber. Deutsch. Math.-Verein., 46 (1936), 26-32.

Received September 25, 2002, and in revised form January 9, 2003, and February 5, 2003.

Online publication October 2, 2003. 